\section{Cave: Katzenhaare im Kino!}

Öffentliche Kinos stehen nicht im Ruf, besonders sauber zu sein. Eine belgische Arbeitsgruppe untersuchte deshalb in sechs großen Kinokomplexen die Belastung von Teppichen und Sitzen mit Bakterien, Endotoxinen, Schimmelpilzen und den Allergenen Der $p$ 1, Bla g 1 und Fel $d$ 1. Die Konzentrationen von Mikroorganismen sowie Kakerlaken- und Hausstaubmilbenallergenen waren insgesamt sehr niedrig, dagegen kamen Katzenallergene in Teppichen in mittlerer und auf Sitzen sogar in hoher Konzentration vor-sie werden offenbar durch die Kleidung der Kinobesucher eingeschleppt.

Nolard N, Beguin H, Symoens F, Chasseur C, Wéry L. Allergens in cinemas. XXII Congress of the EAACI, Paris, 7.-11. Juni 2003

\section{Hunde als Asthmaschutz?}

In einer Studie über 20 Jahre wurden die Daten von 77 Patienten dokumentiert, die vor ihrem drittem Lebensjahr wegen einer Bronchiolitis oder einer Pneumonie stationär behandelt worden waren. 22 von ihnen litten als junge Erwachsene unter Asthma, darunter allerdings keiner, der als Kind Kontakt zu einem Hund gehabt hatte. Tatsächlich schützte

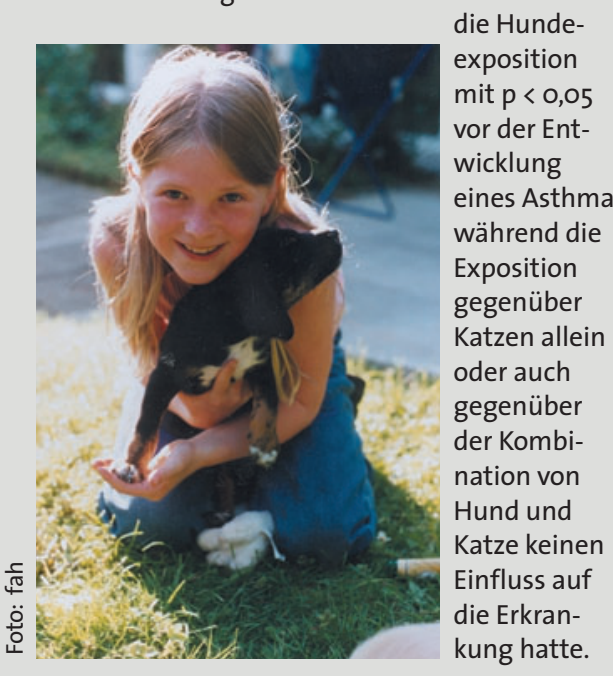

Piippo-Savolainen E, Korhonen $\mathrm{K}$, Remes ST, Kannisto S, Remes K, Korppi $\mathbf{M}$. Does a furred pet at home in early childhood decrease asthma or allergy in early adulthood: a 20-year follow-up study among subjects hospitalised due to low-airway illness in infancy. XXII Congress of the EAACI, Paris, 7.-11. Juni 2003

\section{Congress of the EAACI, Paris 2003}

\section{Evolution der Immuntherapie}

\author{
„Allergie heilen, Asthma verhindern“ - auf diesen Nenner können \\ die Ergebnisse der Studien zur klassischen subkutanen spezifischen \\ Immuntherapie (SIT) gebracht werden. Doch während man beim \\ SIT-Wirkmechanismus auf gesichertem Boden steht, fangen für die \\ sublinguale Immuntherapie (SLIT) die Forschungen erst an.
}

D ie subkutane spezifische Immuntherapie mit standardisierten Allergenextrakten wird mittlerweile seit 20 Jahren praktiziert. Die in dieser Zeit gesammelten Erfahrungen aus Forschung und Klinik fasste Dr. Lars Jacobsen, Hørsholm, Dänemark, zusammen. Die beobachteten Effekte können in vier Bereiche gegliedert werden:

- der frühe Effekt mit sofortiger Reduktion von Symptomen und $\mathrm{Zu}$ satzmedikationen,

- der fortgesetzte Effekt mit weiterer Symptomverbesserung und reduzierter bronchialer Hyperreagibilität im Laufe der Behandlung,

- der Langzeiteffekt mit persistierender Reduktion von Symptomen und Hyperreaktivität auch nach Absetzen der SIT und

- der präventive Effekt mit Reduzierung der Neusensibilisierungen und Hemmung des Etagenwechsels von der Rhinitis zum Asthma.

\section{Hochdosistherapie effektiver}

Die positiven Effekte der Immuntherapie sind abhängig von der (Gesamt-) Dosis und der Therapiedauer: je höher die Dosis und je länger die Therapiedauer, desto besser das Resultat.

Eine solche Dosisabhängigkeit wurde auch in einer aktuellen plazebokontrollierten Studie an 203 Patienten mit allergischer Rhinitis aufgrund einer Sensibilisierung gegen Phleum pratense gezeigt, die von Prof. Dr. Anthony J. Frew, Southampton, Großbritannien, präsentiert wurde. Die Verumpatienten erhielten entweder 100.000 SQ-Einheiten oder 10.000 SQ-Einheiten als Erhaltungsdosis. Das beste Ergebnis - Symptomreduktion und Rückgang an sym- ptomatisch wirkenden Medikamenten erzielte die Hochdosisgruppe vor der Gruppe mit 10.000 Einheiten $(p<0,001$ bzw. $p=0,013$, jeweils gegenüber Plazebo). Auch die Lebensqualität war unter der hohen Dosis besser als unter den 10.000 Einheiten, die Verträglichkeit in beiden Verumgruppen gut.

\section{Unter SLIT steigt spezifisches IgE}

Während die immunologischen Mechanismen bei der SIT gut geklärt sind, ist man bei der SLIT noch auf Vermutungen angewiesen, erklärte Prof. Dr. Christof Ebner, Wien, Österreich. Als Wirkprinzip wird bei dieser Applikationroute eine Beeinflussung des MALT (Mucosa-associated Lymphatic Tissue) mit Induktion einer Toleranz diskutiert. $\mathrm{Ob}$ die Mechanismen der Toleranzbildung bei hoher und niedriger Allergendosis unterschiedlich sind, ist noch nicht geklärt. Fest steht aber, dass nur die nasale und sublinguale Anwendung wirksam sind, eine lokale bronchiale Applikation ist wirkungslos.

Immunologisch findet man bei der SLIT eine angehobene Degranulationsschwelle für Mastzellen und Basophile sowie eine Reduktion von Mediatoren wie Zytokinen und Chemokinen sowie von Entzündungszellen. Doch scheint der zugrundeliegende Mechanismus sich von dem der SIT zu unterscheiden: Das TH1/TH2-Verhältnis wird nicht verändert, das IgG steigt moderat an, aber auch das spezifische IgE - ein Paradoxon, das bisher nicht verstanden ist. $b k$

Symposium „Allergy vaccination: curing allergy - preventing asthma“ (unterstützt von ALK-Abelló). XXII Congress of the EAACI, Paris, 7.-11. Juni 2003 